\title{
Canine Prostate Carcinoma: Four Clinical Cases in Sexually Intact and Neutered Dogs
}

\author{
Enrico Bigliardi, Carla Bresciani, Anna Maria Cantoni, Francesco Di Ianni, Giorgio Morini, \\ Simone Voccia, Attilio Corradi, Enrico Parmigiani \\ Medical Veterinary Sciences Department, University of Parma, Parma, Italy \\ Email: enrico.bigliardi@unipr.it
}

Received September 11, 2012; revised October 12, 2012; accpeted October 19, 2012

\begin{abstract}
Prostate cancer is one of the most important malignancies in men. In old men the frequency of prostate cancer at necroscopy has been reported to exceed $40 \%$. Dogs are the only large mammals other than humans with a significant incidence of spontaneous prostate cancer. Adenocarcinoma, transitional cell carcinoma and undifferentiated carcinoma are the most common histological type but the precise cell of origin in dog is not known. The incidence of prostatic carcinoma in dogs is low $(0.2 \%-0.6 \%)$. Prostatic carcinomas occur in sexually intact and neutered dogs and the risk increase in castrated dogs associated to pulmonary and bone metastases. The castration does not initiate the development of prostatic carcinoma in dog but does favour tumour progression. In men the early stage detection of prostate cancer can offer various therapies as radical prostatectomy, radial therapy, thermal ablation, anti-androgen therapy, chemotherapy. In dogs the diagnosis is often in advanced stage of the cancer and the survival time for dogs with prostate cancer is poor. The median time reported is 30 days after diagnosis. In this study we reported three cases of prostatic carcinoma in intact sexually dogs and one in a neutered dog. The sexually intact subjects were older (mean age $=10.5$ years) and they had prostatic adenocarcinoma (PCA). The interval between castration and onset of prostatic problems was 3 years. All the dogs showed dysuria, macroscopic hematuria, dyschezia and ataxia. All dogs have been euthanized in order to relieve pain and suffering.
\end{abstract}

Keywords: Prostate; Carcinoma; Neutered Dog

\section{Introduction}

In human, dog and cat the prostate is a bilobed retroperitoneal gland, encircling the neck of the urinary bladder and the proximal urethra. The normal prostate is an exocrine gland with a tubuloalveolar structure, composed of a glandular component surrounded by a smooth muscle and connective tissue stroma. Regarding the vascular anatomy of the canine prostate gland, each prostatic lobe has an independent vascularization. The blood supply to each lobe is from the homolateral prostatic artery. Three small vessels arise from the prostatic artery (cranial, middle, and caudal) through the prostate gland. The prostatic artery is small, with a wall structure similar to muscular arteries. The prostatic vein is a small-size valved vein. The canine prostate gland has capsular, parenchymal and urethral vascular zones. The surface vessels of the capsule are predominantly veins and the diameter of the arterial vessels is larger than in veins. Trabecular vessels can be of two types: direct and branched. The prostatic parenchyma is supplied by trabecular vessel branches. Periacinary capillaries are fenestrated and form a circular net. The processes of myoepithelial cells embrace both the acinar and periacinar capillaries. The prostatic part of the urethra is supplied by an independent branch of the prostatic artery. The prostatic urethral part is drained by the prostatic vein, the vein of the urethral bulb and the ventral prostate veins. Urethralis muscles begin in the urethral prostatic part $[1,2]$.

Prostate cancer is one of the most important malignancies in men. In old men the frequency of prostate cancer at autopsy has been reported to exceed $40 \%$ [3]. Dogs are the only large mammals other than humans with a significant incidence of spontaneous prostate cancer [4].

Adenocarcinoma, transitional cell carcinoma and undifferentiated carcinoma are the most common histological type [5], but the precise cell of origin in dog is not know. The incidence of prostatic carcinoma (PCA) in dogs is low $(0.2 \%-0.6 \%)$ in necroscopy studies [6], but it shares several of the features of the disease in humans [7]. Tumour growth outside the prostate is common in dog and frequently there are pulmonary a bone metastases [6]. The prostate is an androgen-dependent gland. Or- 
chiectomy result in regression of prostatic volume in dog with prostatic hyperplasia (BPH) and androgen administration to neutered dogs causes hypertrophy of the gland [8]. Therefore it is surprising to find a high prevalence of prostatic carcinoma in neutered dog [6]. In dog prostatic cancer is an aggressive Tumour that carries a poor prognosis.

In this study we reported four cases of prostate cancer in sexually intact (3) and neutered (1) dogs.

\section{Materials and Methods}

Three mixed-breed and one Border Collie adult male dogs were admitted at the Teaching Veterinary Hospital of Medical Veterinary Science Department of University of Parma with clinical sings of prostatic disease. Age ranged between 6 and 12 years (mean age 9.25). Body weight ranged between $22 \mathrm{~kg}$ and $35 \mathrm{~kg}$ (mean $26 \mathrm{~kg}$ ) (Table 1). The dimensions and the aspect of the prostate gland were quantified using conventional ultrasonography. The diagnosis was made by means of cytological echoguided fine-needle aspiration biopsies of prostate. One dog (Border Collie) was neutered and the other were sexually intact. All animals were premedicated with butorphanol $(0.2 \mathrm{mg} / \mathrm{kg})$ (Dolorex ${ }^{\circledR}$, Intervet, Italy) and dexmedetomidine $(5 \mu \mathrm{g} / \mathrm{kg})$ (Dex-domitor ${ }^{\circledR}$, Pfizer, Italy) intramuscolary and $\mathrm{NaCl} 0.9 \%(4 \mathrm{ml} / \mathrm{kg} / \mathrm{h})$ were administered IV during procedures. General anaesthesia was induced and maintained with propofol (4 mg/kg) (Rapinovet $^{\circledR}$, Schering-Plough Animal Health). Dogs were monitored for cardiac and respiratory rate and during recovery from anaesthesia. Ultrasonographic examination of the prostate gland was performed using a MyLab 30 Gold (Esaote, Genoa, Italy) scanner. To limit the color-blooming artifact the pulse repetition frequency and gain were adjusted before starting the examination. Imaging was performed with a $5-7.5 \mathrm{MHz}$ phase-array transducer. Settings for color and power Doppler were constant for all examinations (color gain 60\%, medium wall filter, pulse repetition frequency $700 \mathrm{~Hz}$, image depth $5-7 \mathrm{~cm}$ ). For Doppler imaging, a sample volume was placed over the prostate gland. All imaging parameters were constant during the procedure. Doppler images

Table 1. Age and weight of dogs.

\begin{tabular}{ccc}
\hline Dog & Weight & Age \\
\hline 1 & 25 & 6 \\
2 & 18 & 9 \\
3 & 26 & 10 \\
4 & 35 & 12 \\
Mean & 26 & 9.25 \\
\hline
\end{tabular}

were captured for analysis using ImageJ (National Institute of Health USA, on-line version). A region of interest (ROI) was drawn around the prostate gland without including adjacent tissue. Three measurements were computed for each ROI. We calculated the fractional area which represents a vascularity index, showing the percentage area of the prostate gland occupied by blood vessels [2]. Systolic and diastolic peak velocity, enddiastolic velocity, pulsatility index and resistive index were also computed.

Necroscopy, after euthanasia $\left(\right.$ Tanax $\left.^{\circledR}\right)$, was performed and specimens were collected from urethra, prostate gland and iliac lymph-nodes as well as organ, apparatus and system of abdominal, thoracic and neurocranic cavities. Specimens were immediately fixed in buffered phosphate formalin $10 \% \mathrm{v} / \mathrm{v}$. Formalin-fixed-paraffin embedded $5 \mu \mathrm{m}$ thick sections were stained for routine diagnostic histology (H\&E) and studied using a conventional microscope Eclipse E 800 Nikon. Images were captured using a Nikon digital sight DS-L2 system.

\section{Results}

In all the dogs were diagnosed prostate diseases. The sexually intact dogs were older (mean age $=9.25$ years) and they had prostatic adenocarcinoma (PCA). The interval between castration and onset of prostatic problems was 3 years. All the dogs had dysuria, macroscopic hematuria, dyschezia and ataxia. The ultrasonographic examination shown increase of the volume (mean $6 \mathrm{~cm}$ ) with foci of necrosis (ipoechoic) and iperechoic areas (Figures 1 and 2). The capsular limit is not well defined. Systolic and diastolic peak velocity, the end-diastolic velocity, resistive index and pulsatility index values were increased in accordance with other published values [9]. Doppler examination shows increased blood flow within prostate with high fractional area index [2]. The neutered dog was 6 years old and had PCA associated to prostatic cyst (Figure 3), pulmonary and bone metastases. Two sexually intact dogs presented rectal tenesmus due to enlargement of prostate. The third dog had neoplastic invasion in the urinary bladder and in the perineural space and pelvic musculature accompanied by posterior paresis. In the final stage of disease lethargy, weight loss, anorexia and poor body condition were presenting all patients. The Border Collie had dysuria till anuria due to local invasion into the prostatic urethra with partial obstruction, and for the last month of life it has been catheterized using a Foley's Catheter inserted into bladder via the urethra to allows the patient's urine to drain. All dogs have been euthanized in order to relieve pain and suffering. In neutered dog prostate was enlarged for a presence of neoformation sized $4.5 \times 5.5 \mathrm{~cm}$. Rectum, in the pelvic canal, was compressed by newly formed mass. The proximal tract of pelvic urethra was stenosed by neo- 


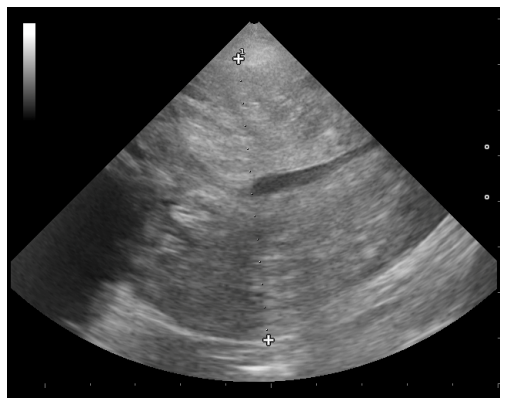

Figure 1. Prostate carcinoma: increase of volume and change of normal echographic findings.

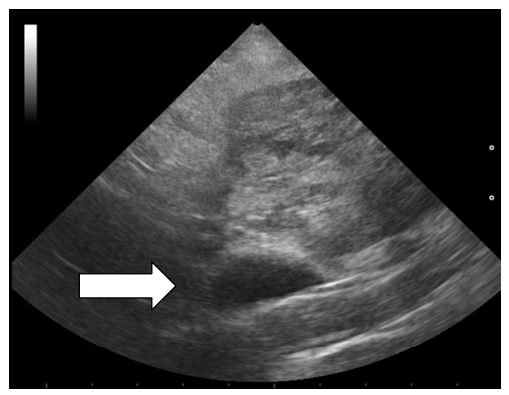

Figure 2. Prostate carcinoma: in the bottom of the image is present one para-prostatic cyst (white arrow). Note the increase of volume and alteration of prostate tissue.

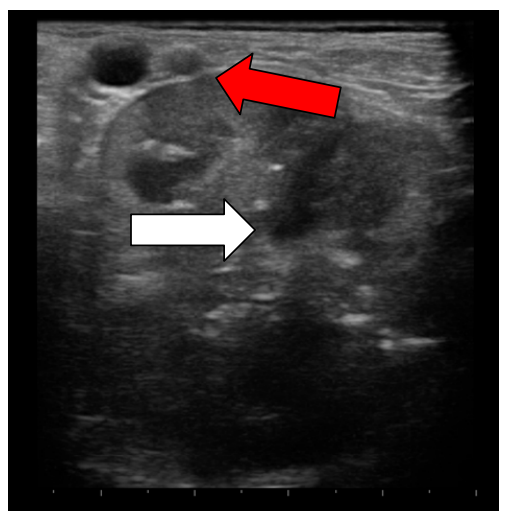

Figure 3. Prostate carcinoma: Neutered dog. The structure of prostate shows ipoechoic (white arrow) and anechoic areas (red arrow).

formed tissue or involvement of the urethral disseminated prostate. Urinary bladder was distended for urinary stasis. Foci of necrosis were observed on cut surface of prostate neoformation. No evidence of gross pathology was observed on iliac lymph-nodes. The largest one sized $1 \times 1.5 \mathrm{~cm}$, were recorded in lung parenchyma. A voluminous newly formed mass involved also the 7th thoracic vertebra. At histopathology a typical neoplastic malignant hystotype classified as adenocarcinoma of the prostate was diagnosed in the mass recorded in prostate gland and urethral disseminated prostate, in the pulmonary and vertebral metastasis (Figures 4-7).

\section{Discussion and Conclusions}

Prostatic carcinoma is a definition with different meanings and mostly the neoplasia is adenocarcinoma (prostate glandular tissue) as in human. In dogs we found adenocarcinoma, transitional cell carcinoma, mixed carcinoma and squamous cell carcinoma. Prostatic carcinomas occur in sexually intact and neutered dogs and pulmonary and bone metastases are common [6]. Some Authors reported that the risk to be associated to pulmonary

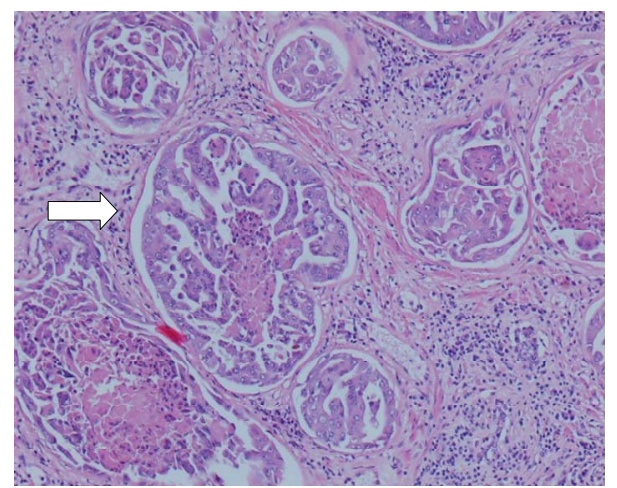

Figure 4. Dog prostate gland in neutered dog: neoplastic cells arranged in irregular tubules $(H \& E$ 10 $\times)$.

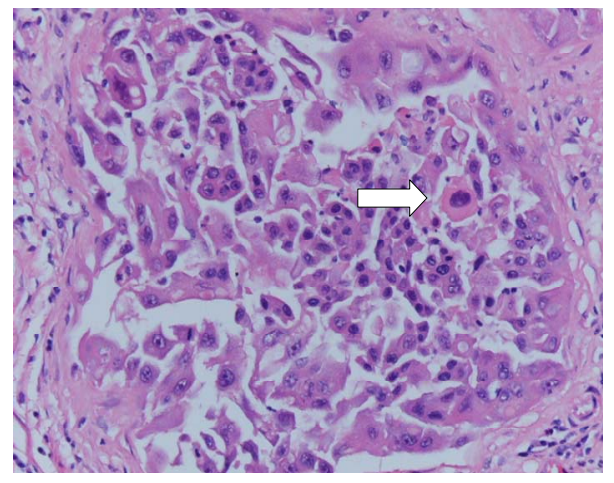

Figure 5. Dog prostate gland in neutered dog: neoplastic cells with marked atypical features (white arrow, $H \& E$ 20×).

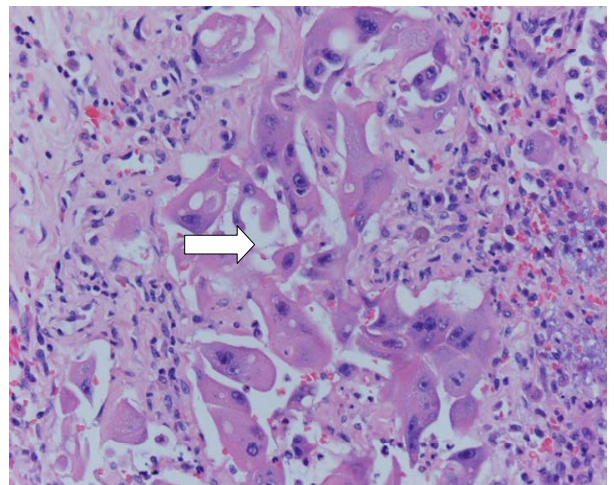

Figure 6. Dog urethra of neutered dog: metastatic neoplastic cells into the lumen (white arrow, H\&E 20×). 


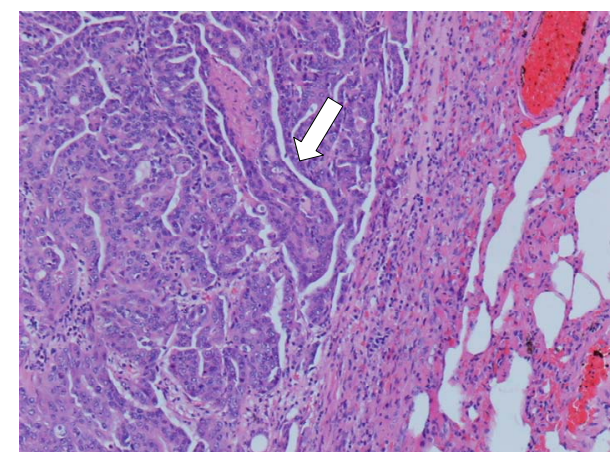

Figure 7. Dog lung metastatic neoplastic cells arranged in irregular tubules and solid cords (white arrow H\&E 10×).

and bone metastases increase in castrated dogs [4]. The frequency of prostatic carcinoma in dog is low and the prevalence reported from necroscopy studies ranges between $0.2 \%$ and $0.6 \%$ [6]. The prevalence of PCA in neutered dogs is not well cleared. The castration does not initiate the development of prostatic carcinoma in dog but does favour Tumour progression [3]. Human and canine prostate cancer had important difference: human prostate neoplasia is highly dependent upon androgen (growth factor), in fact in the men androgen deprivation is a good therapy for prostate cancer. In human males there are pre-malignant marker lesions with risk factors such as familial, diet, inflammation disease, in dog the pre-malignant lesions are uncommon and castration is a risk factor for prostate cancer development. In men most prostate cancer are latent; in dog the aggressiveness of prostate cancer is high. The early stage of human prostate cancer is an intra-epithelial neoplasia (PIN) in dog the relationship between PIN and prostate carninoma is unknown. Canine prostate neoplasia does not express androgen receptor and the castration may be associated with an increased risk of prostate adenocarcinoma $[4,10]$. Castration modified the stromal component of the gland from primarily actin-positive smooth muscle cells to vimentin-positive mesenchymal cells. This condition could play a role in the development of prostate cancer in neutered dogs [11]. Human prostatic carcinoma arise in the peripheral zone, in dog is unclear but there is evidence that the neoplasia may stem from ductular epithelium adjacent to the periurethral zone [4]. Human carcinoma is graded using the Gleason system that is a highly accurate predictor of disease progression [4]. Much less is known regarding the pathogenesis and definitive origin of canine prostatic neoplasia. Neoplastic canine prostate frequently contain foci of benign hyperplasia (BPH), cyst (Border Collie) and inflammation. Survival time for dogs with prostate cancer is poor. The median time reported is 21 days [12] and 30 days [4] and most of patients were euthanized at the time of diagnosis. In our cases Border Collie were euthanized two month after cytological di- agnosis of carcinoma. In men the early stage detection of prostate cancer can offer various therapies as radical prostatectomy, radial therapy, thermal ablation, anti- androgen therapy, chemotherapy. In dogs the diagnosis is often in advanced stage of the cancer. At this time is not universally accepted the prostatectomy because the prostate cancer is associated with a high rate to metastasis and the surgical procedure has a significant postoperative morbidity and is not clear that this procedure will improve survival [4]. Complication of radiation therapy as chronic colitis, gastrointestinal perforation, necrotic ulceration and chronic cystitis have been reported [13]. It is evident that prostate cancer in dog is aggressive with poor prognosis. We think that when the dogs have advanced stage of benign prostatic hyperplasia before castration could useful performed prostatic biopsies to confirm the absence of cancer because the orchiectomy can favour cancer progression.

\section{REFERENCES}

[1] M. Stefanov, "Extraglandular and Intraglandular Vascularization of Canine Prostate," Microscopy Research and Technique, Vol. 64, No. 3, 2004, pp. 188-197.

[2] E. Bigliardi and L. Ferrari, "Contrast-Enhanced Ultrasound of the Normal Canine Prostate Gland," Veterinary Radiology Ultrasound, Vol. 52, No. 1, 2011, pp. 107-110.

[3] E. Teske, E. C. Naan, E. M. Van Dijk, E. Van Garderen and J. A. Schalken, "Canine Prostate Carcinoma: Epidemiological Evidence of an Increased Risk in Castrated Dogs," Molecular and Cellular Endocrinology, Vol. 197, No. 1-2, 2002, pp. 251-255.

doi:10.1016/S0303-7207(02)00261-7

[4] B. E. LeRoy and N. Northrup, "Prostate Cancer in Dogs: Comparative and Clinical Aspects," The Veterinary Journal, Vol. 180, No. 2, 2009, pp. 149-162. doi:10.1016/j.tvj1.2008.07.012

[5] K. K. Cornell, D. G. Bostwick, D. M. Cooley, G. Hall, H. J. Harvey, M. J. Hendrick, B. U. Pauli, J. A. Render, G. Stoica, D. C. Sweet and D. J. Waters, "Clinical and Pathologic Aspects of Spontaneous Canine Prostate Carcinoma: A Retrospective: An Aalysis of 76 Cases," The Prostate, Vol. 45, No. 2, 2000, pp. 173-183. doi:10.1002/1097-0045(20001001)45:2<173::AID-PROS $12>3.0 . \mathrm{CO} ; 2-\mathrm{R}$

[6] F. W. Bell, J. S. Klausner, D. W. Hayden, D. A. Feeney and S. D. Johnston, "Clinical and Pathological Features of Prostatic Adenocarcinoma in Sexually Intact and Castrated Dogs: 31 Cases (1970-1987)," Journal of the American Veterinary Medical Association, Vol. 199, No. 11, 1991, pp. 1623-1630.

[7] A. Maini, C. Archer, C. Y. Wang and G. P. Haas, "Comparative Pathology of Bening Prostatic Hyperplasia and Prostate Cancer," In Vivo, Vol. 11, No. 4, 1997, pp. 293300.

[8] W. Mahapokai, Y. Xue, E. van Garderen, F. J. van Sluijs, J. A. Mol and J. A. Schalken, "Cell Kinetics and Differ- 
entiation after Hormonal-Induced Prostatic Hyperplasia in the Dog," Prostate, Vol. 44, No. 1, 2000, pp. 40-48. doi:10.1002/1097-0045(20000615)44:1<40::AID-PROS6 $>3.0 . \mathrm{CO} ; 2-\mathrm{J}$

[9] A. R. Gunzel-Apel, C. Moehrke and C. P. Nautrup, "Colour-Codex and Pulsed Doppler Sonography of Canine Testis, Epididymis and Prostate Gland: Physiological and Pathological Findings," Reproduction in Domestic Animals, Vol. 36, No. 5, 2001, pp. 236-240. doi:10.1046/j.1439-0531.2001.00288.x

[10] K. U. Sorenmo, M. Goldschmidt, F. Shofer, C. Goldkamp and J. Ferracone, "Immunohistochemical Characterization of Canine Prostatic Carcinoma and Correlation with Castration Status and Castration time," Veterinary and Comparative Oncology, Vol. 1, No. 1, 2003, pp. 48-56. doi:10.1046/j.1476-5829.2003.00007.x
[11] F. R. Shidaifat, M. Daradka and R. A. Omari, "Effect of Androgen Ablation on Prostatic Cell Differentiation in Dogs," Endocrine Research, Vol. 30, No. 3, 2004, pp. 327-334. doi:10.1081/ERC-200033196

[12] K. U. Sorenmo, M. Goldschmidt, F. Shofer, C. Goldkamp and J. Ferracone, "Evaluation of Cyclooxigenase-1 and Cyclooxygenase-2 Expression and the Effect of Cycloxygenase Inhibitors in Canine Prostatic Carcinoma," Veterinary and Comparative Oncology, Vol. 2, No. 1, 2004, pp. 13-23.

[13] J. J. Arthur, M. M. Kleiter, D. E. Thrall and A. F. Pruitt, "Characterization of Normal Tissue Complications in 51 Dogs Undergoing Definitive Pelvic Region Irradiation," Veterinary Radiology and Ultrasound, Vol. 49, No. 1, 2008, pp. 85-89. doi:10.1111/j.1740-8261.2007.00322.x 